\title{
ChemComm
}

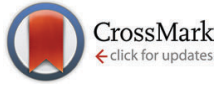

Cite this: Chem. Commun., 2015, 51, 13764

Received 16th March 2015,

Accepted 21st July 2015

DOI: $10.1039 / \mathrm{c} 5 \mathrm{cc} 02218 \mathrm{e}$

www.rsc.org/chemcomm

\section{Are metal-free pristine carbon nanotubes electrocatalytically active $+\dagger$}

\author{
Yi Cheng, Jin Zhang and San Ping Jiang*
}

\begin{abstract}
Metal-free (i.e., residual metallic impurities-blocked) carbon nanotubes (CNTs) do show electrocatalytic activity for $\mathrm{H}_{2}$ evolution, $\mathrm{O}_{2}$ evolution and $\mathrm{O}_{2}$ reduction reactions (HER, OER \& ORR) in alkaline solutions, but their activities strongly depend on the number of walls or inner tubes with a maximum for CNTs with 2-3 walls.
\end{abstract}

The electrochemistry of carbon based materials such as carbon nanotubes (CNTs) plays an extremely important role in the electrochemical energy conversion and storage technologies such as fuel cells, advanced batteries, water electrolysis and supercapacitors. CNTs possess unique properties such as large specific surface area, excellent chemical and mechanical properties, structural stability and high electrical conductivity and have been extensively used as electrocatalyst supports for fuel cells, water electrolysis, etc. ${ }^{1,2}$ However, despite the vital role of CNTs in electrocatalysis, there are considerable uncertainties regarding the origin of electrocatalytic activity of CNTs. This is largely due to the fact that CNTs are commonly synthesized using metal containing catalysts and inevitably there are a significant amount of residual metallic impurities within the CNTs such as $\mathrm{Fe}, \mathrm{Co}, \mathrm{Ni}, \mathrm{Mn}$, etc. ${ }^{3}$ Kruusenberg et al. studied the electrocatalytic activity of acid-treated single-walled and multiwalled CNTs (SWNTs \& MWNTs) in acid solution and found that the acid-treated CNTs are less active catalysts for ORR as compared to as-received CNTs. ${ }^{4}$ The same group also indicated that CNTs show a higher activity for ORR in alkaline solutions but the metal impurities only play a minor role in the solution of high pH. ${ }^{5,6}$ Matter et al. synthesized nitrogen-doped CNTs via pyrolysis of acetonitrile at $900{ }^{\circ} \mathrm{C}$ using Fe particles supported on alumina as a catalyst and tested with respect to the ORR. ${ }^{7}$ It was concluded that the role of the Fe particles is likely that of a catalyst for both the CNT growth and the formation of catalytically more active carbon nanostructures during pyrolysis, but not as an active site

Fuels and Energy Technology Institute \& Department of Chemical Engineering, Curtin University, Perth, WA 6102, Australia. E-mail: s.jiang@curtin.edu.au

$\dagger$ Electronic supplementary information (ESI) available: Experimental section, TEM, ICP, Raman, BET, XPS, and supplementary results and discussion. See DOI: $10.1039 / \mathrm{c} 5 \mathrm{cc} 02218 \mathrm{e}$ for the ORR. ${ }^{7}$ Wang and Pumera studied the ORR on three different MWNTs with different levels of metal impurities and the results show that $\mathrm{Ni}$, Co and Fe oxides are not active for ORR and the only active metal impurity for ORR in $\mathrm{KOH}$ solution is $\mathrm{MnO}_{2}$. Based on these results, the author concluded that pure CNTs do not exhibit any electrocatalytic effect on ORR and that the metallic impurities are the ones truly responsible for the electrocatalytic reduction of $\mathrm{O}_{2}$, and not inherent CNT materials themselves. ${ }^{8}$ On the other hand, Byers et al. showed that the electrochemical activity on straight and defect-free SWNTs for ORR is comparable to Au, based on the scanning electrochemical cell microscopy study on well-defined SWNTs and concluded that pristine SWNTs are not inert: they exhibit substantial intrinsic electrochemical activity, which needs to be taken into account in directing their use in the future. ${ }^{9}$

The considerable discrepancies related to the origin and nature of electrocatalytic activity of CNTs may be related to the intrinsic properties of CNTs. We recently studied the electrocatalytic activity of pristine CNTs and showed that CNTs composed of 2-7 concentric tubes or walls have significantly higher electrochemical activities for the OER in alkaline solutions as compared with typical SWNTs and MWNTs. ${ }^{10}$ The results indicate that the electrocatalytic activity of CNTs shows a distinct volcano-type curve as a function of the number of walls of CNTs. We utilized Pt and Pd nanoparticles as model catalysts for methanol, ethanol and formic acid oxidation, and demonstrated that the number of walls of CNTs plays a critical role in promoting the electrocatalytic activity of Pt and Pd and the distinctive volcano-type curves are also observed. ${ }^{11,12}$ However, the effect of metallic impurities could not be convincingly ruled out due to the fact that there are significant metal impurities within the CNTs used. ${ }^{10}$

Here, we carefully designed the experiments to produce metal-free CNTs. CNTs with different number of walls and diameters were selected and treated using a relatively mild purification procedure in order to avoid the structural damage to the CNTs. ${ }^{10}$ Based on the number of walls, the CNTs were characterized as belonging to one of the seven groups labelled as CNT- $n$ for $n=1-7$ (Fig. S1-S3, ESI $\dagger$ ). CNT- 1 composed of $79 \%$ 
SWNTs with OD $=1.97 \mathrm{~nm}$. CNT-2 is mainly double-walled CNTs (DWNTs, 65\%) with OD $=3.30 \mathrm{~nm}$. CNT-3 and CNT-4 are mainly triple-walled CNTs (TWNTs, 52-57\%) with OD $=3.80$ and $4.00 \mathrm{~nm}$, respectively. The average number of walls of CNT-5 is seven with OD $=5.10 \mathrm{~nm}$. CNT-6 and CNT-7 are typical MWNTs with the number of walls over 12 and 20 and $\mathrm{OD}=13.90$ and $20-40 \mathrm{~nm}$, respectively. The amount of metal impurities was in the range of $0.4-1.3 \%$ as determined by the ICP-OES analysis and mainly consisted of Fe, Co, Mo and Ni (Table S1, ESI $\dagger$ ). To specifically poison and completely block the effect of residual metallic impurities on the sidewalls of CNTs, we employed $\mathrm{KCN}$. Cyanide, $\mathrm{CN}^{-1}$ ions are known to coordinate strongly with transition metals such as Fe, $\mathrm{Co}$ and $\mathrm{Ni}$ in the axial position, poisoning and blocking the interaction of reactants such as $\mathrm{O}_{2}$ with the metal. ${ }^{13,14}$ We validated the KCN method by artificially depositing $3.5 \%$ of NiCo catalysts onto CNT-7 (3.5\% NiCo/CNT-7). The results indicate that $3.5 \% \mathrm{CoNi} /$ CNT-7 is active for OER, exhibiting a current of $27 \mathrm{~mA} \mathrm{~cm}^{-2}$ at $1.8 \mathrm{~V}$ in $1 \mathrm{M} \mathrm{KOH}$ (Fig. S5, ESI $\dagger$ ). However, in the presence of $10 \mathrm{mM} \mathrm{KCN}$, the current density at $1.8 \mathrm{~V}$ is reduced significantly to $\sim 3 \mathrm{~mA} \mathrm{~cm}^{-2}$, which is the same as that of pristine CNT-7. This confirms that 10 $\mathrm{mM} \mathrm{KCN}$ in $1 \mathrm{M} \mathrm{KOH}$ solution can completely block the activity of at least $3.5 \mathrm{wt} \%$ of NiCo metal catalysts on CNTs. Please note that the maximum residual metal impurities in the CNT samples used in the study are $\sim 1.3 \%$, much lower than $3.5 \mathrm{wt} \%$ used in the validity test.

The electrochemical activities of CNTs were studied in both $1 \mathrm{M}$ $\mathrm{KOH}$ and $1 \mathrm{M} \mathrm{KOH}+10 \mathrm{mM}$ KCN solutions for OER, HER and ORR, the most important reactions in the electrochemical energy conversion and storage. Fig. 1 shows the linear scan voltammetry (LSV) curves of CNTs for OER. In $1 \mathrm{M} \mathrm{KOH}$, the electrocatalytic activity (i.e., current density, $j$ measured at $1.8 \mathrm{~V} v$ s. RHE) of CNTs varies significantly with the number of walls of CNTs, showing a characteristic of volcano curves as a function of number of walls (Fig. 1A and B). The best activity was observed on CNT-2, CNT-3 and CNT-4, consistent with previous results. ${ }^{10}$ With the addition of $10 \mathrm{mM} \mathrm{KCN}$ in $1 \mathrm{M} \mathrm{KOH}$ solution, the activity of CNTs for OER is substantially decreased (Fig. 1C). For example, in the case of TWNTs, CNT-3, $j$ is $\sim 30 \mathrm{~mA} \mathrm{~cm} \mathrm{~cm}^{-2}$ at $1.8 \mathrm{~V}$ for OER, much lower than $56 \mathrm{~mA} \mathrm{~cm} \mathrm{~cm}^{-2}$ measured under identical conditions in the

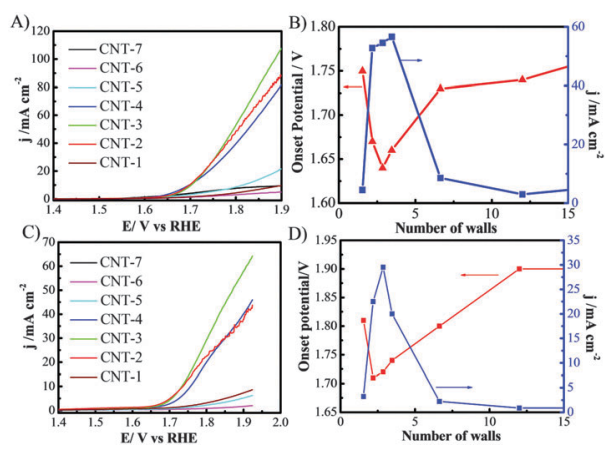

Fig. 1 (A and B) LSV curves and the corresponding plots of $j$ measured at $1.8 \mathrm{~V}$ and onset potential as a function of the number of walls of CNTs for OER in $1 \mathrm{M} \mathrm{KOH}$; ( $C$ and D) LSV curves and corresponding plots of $j$ measured at $1.8 \mathrm{~V}$ and onset potential as a function of the number of walls of CNTs for OER in $1 \mathrm{M} \mathrm{KOH}+10 \mathrm{mM} \mathrm{KCN}$. Scan rate $=10 \mathrm{mV} \mathrm{s}^{-1}$, rotating rate $=1600 \mathrm{rpm}$ and CNTs loading $=0.05 \mathrm{mg} \mathrm{cm}^{-2}$. absence of KCN. The onset potential is $\sim 1.67 \mathrm{~V}$, also higher than $1.64 \mathrm{~V}$ in the absence of $\mathrm{KCN}$. The significant reduction in the activity of CNT-3 in $1 \mathrm{M} \mathrm{KOH}+10 \mathrm{mM} \mathrm{KCN}$ indicates that the addition of $\mathrm{KCN}$ is very effective to completely poison and block the active centre of metallic impurities for OER (see also Fig. S5, $\mathrm{ESI} \dagger$ ), similar to that reported by Thorum et al. on the blocking effect of the iron sites by $\mathrm{CN}^{-1} \cdot{ }^{14}$ As shown in Table S1 (ESI $\dagger$ ), CNT-3 contains 5700 ppm Co, 2300 ppm Fe, 2700 ppm Mo and 300 ppm Ni. However, the reduction in the activity appears to be related to CNTs. In the case of MWNTs, CNT-7, the onset potential for OER is $1.79 \mathrm{~V}$ in the presence of KCN (Fig. 1C), similar to that in the absence of KCN, $1.78 \mathrm{~V} . j$ at $1.8 \mathrm{~V}$ is $\sim 1 \mathrm{~mA} \mathrm{~cm}^{-2}$, also close to $\sim 3 \mathrm{~mA} \mathrm{~cm}^{-2}$ for the reaction in the absence of $\mathrm{KCN}$, despite the fact that CNT-7 contains considerable metallic impurities, 2900 ppm Fe, 4900 ppm Mo and 1700 ppm Ni. Despite the reduction in the electrocatalytic activity, the variation of the activity of CNTs for OER in the presence of $\mathrm{KCN}$ is almost identical to that in the absence of KCN, showing a distinctive volcano-type dependence on the number of walls of CNTs (Fig. 1D). In both cases, the most active CNTs for the OER in alkaline solution are the ones with 2-3 walls.

Similar to that observed on OER, the number of walls of CNTs also have significant influence on the HER and ORR in $1 \mathrm{M} \mathrm{KOH}$ in the absence and presence of KCN (Fig. 2). In the case of HER in $1 \mathrm{M} \mathrm{KOH}$ solutions, the electrocatalytic activity of CNTs changes
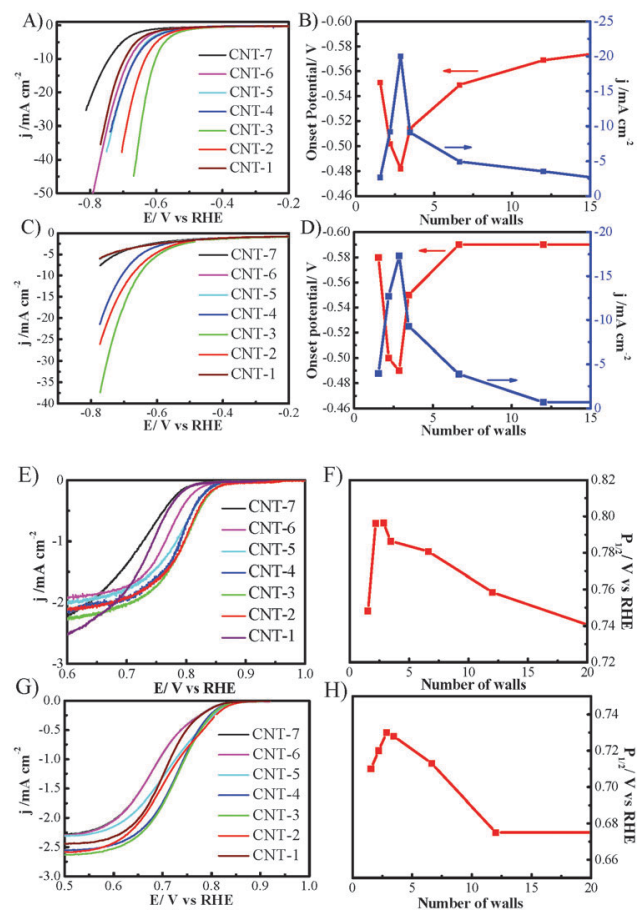

Fig. 2 (A and B) LSV curves and the corresponding plots of $j$ measured at $-0.6 \mathrm{~V}$ and onset potential as a function of the number of walls of CNTs for HER in $1 \mathrm{M} \mathrm{KOH}$; ( $C$ and D) LSV curves and corresponding plots of $j$ measured at $-0.7 \mathrm{~V}$ and onset potential as a function of the number of walls of CNTs for HER in $1 \mathrm{M} \mathrm{KOH}+10 \mathrm{mM} \mathrm{KCN}$; (E and F) LSV curves and corresponding plots of $P_{1 / 2}$ as a function of number of walls of CNTs for ORR in $1 \mathrm{M} \mathrm{KOH}$; ( $\mathrm{G}$ and $\mathrm{H}$ ) LSV curves and corresponding plots of $P_{1 / 2}$ as a function of the number of walls of CNTs for ORR in $1 \mathrm{M} \mathrm{KOH}+10 \mathrm{mM} \mathrm{KCN}$. Scan rate $=10 \mathrm{mV} \mathrm{s}^{-1}$, rotating rate $=1600 \mathrm{rpm}$ and CNTs loading $=0.05 \mathrm{mg} \mathrm{cm}^{-2}$. 
significantly with the number of walls of CNTs with the optimum results observed on CNT-3 (Fig. 2A). With the addition of $10 \mathrm{mM}$ $\mathrm{KCN}$, the activity is substantially reduced, similar to that observed for OER (Fig. 2C). For example, $j$ measured at $-0.6 \mathrm{~V}$ on CNT-3 is $5.3 \mathrm{~mA} \mathrm{~cm}^{-2}$, much lower than $21.0 \mathrm{~mA} \mathrm{~cm}^{-2}$ measured on CNT-3 in $1 \mathrm{M} \mathrm{KOH}$ under identical conditions. On the other hand, the activity of CNTs for HER shows characteristic volcano curves in both the $1 \mathrm{M} \mathrm{KOH}$ and $1 \mathrm{M} \mathrm{KOH}+10 \mathrm{mM}$ KCN solutions (Fig. 2B and D). Identical phenomena were also observed for the ORR on CNTs. The half-wave potential $\left(P_{1 / 2}\right)$ for ORR on CNTs varies significantly with the number of walls in $1 \mathrm{M} \mathrm{KOH}$ and in $1 \mathrm{M}$ $\mathrm{KOH}+10 \mathrm{mM} \mathrm{KCN}$ solutions. In the case of $1 \mathrm{M} \mathrm{KOH}, \mathrm{CNT}-2$ and CNT-3 have the best activity for ORR, exhibiting $P_{1 / 2}$ of $0.80 \mathrm{~V}$, 50 and $80 \mathrm{mV}$ more positive as compared to $0.75 \mathrm{~V}$ on CNT-1 and $0.72 \mathrm{~V}$ on CNT-7, respectively (Fig. 3E). Similar results were also obtained on CNTs with $2-3$ walls for ORR in $1 \mathrm{M} \mathrm{KOH}+10 \mathrm{mM}$ KCN solutions (Fig. $2 \mathrm{G}$ ) even though the $P_{1 / 2}$ values are negatively shifted by $40-80 \mathrm{mV}$. The electrocatalytic activity of CNTs for ORR as measured by the half-wave potentials shows identical volcanotype relationship as a function of number of walls in the presence and absence of $10 \mathrm{mM} \mathrm{KCN}$ in $\mathrm{KOH}$ solution (Fig. $2 \mathrm{~F}$ and $\mathrm{H}$ ).

Due to the effective poisoning and blocking effect of KCN on the active sites of metallic catalysts, the differences in the activity of CNTs with and without addition of KCN can be taken as the electrocatalytic activity of residual metal impurities within CNTs. Fig. 3 shows the plots of the differences in the activity of CNTs for OER, HER and ORR in the absence and presence of KCN. In the case of OER, the impurities show significant catalytic activity and CNT-2 shows the highest electrocatalytic activity of residual metal impurities for OER. This is clearly due to the high content of metal impurities in CNT-2, 13800 ppm. The electrocatalytic activity of metal impurities within CNTs generally shows a correlation with the concentration of the impurities with the exception of MWNTs, e.g., CNT-5, CNT-6 and CNT-7. For example, CNT-5 contains 6550 ppm impurities, similar to the value of $7700 \mathrm{ppm}$ in CNT-4. However, the activity of impurities of CNT-5 is $7.5 \mathrm{~mA} \mathrm{~cm}{ }^{-2}$, significantly lower than $20.1 \mathrm{~mA} \mathrm{~cm}{ }^{-2}$ measured on CNT-4. In the case of CNT-7, the impurity content is very high, $9500 \mathrm{ppm}$, but the activity is

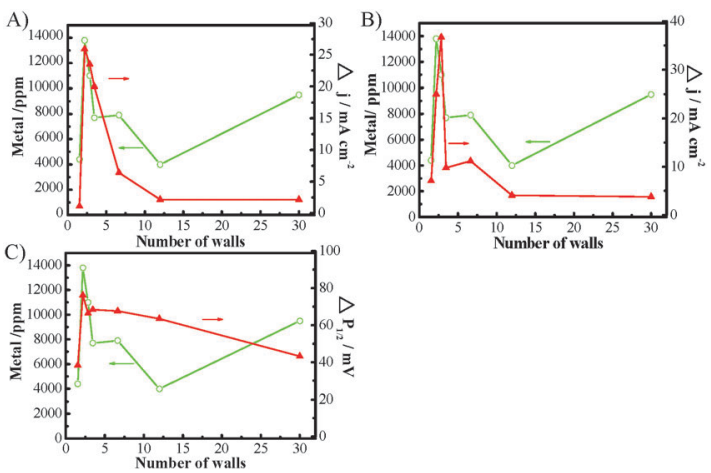

Fig. 3 (A) Plots of the differences in the electrocatalytic activity of CNTs for (A) OER, (B) HER and (C) ORR, measured in $1 \mathrm{M} \mathrm{KOH}$ and $1 \mathrm{M} \mathrm{KOH}+10 \mathrm{mM}$ $\mathrm{KCN}$ solutions. $\Delta j=j_{\mathrm{KOH}}-j_{\mathrm{KOH}+\mathrm{KCN}}$, where $j$ was measured at $1.8 \mathrm{~V}$ for OER

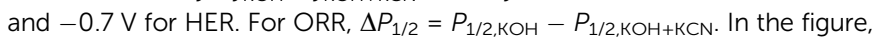
the total residual metallic impurities in ppm are also given. negligible, $2.8 \mathrm{~mA} \mathrm{~cm}^{-2}$ for OER measured at $1.8 \mathrm{~V}$ (Fig. 3A). Similar results were also obtained on the electrocatalytic activity of metal impurities within CNTs for HER and ORR. This indicates that the electrocatalytic activity of residual metallic impurities only becomes pronounced on CNTs with 2-3 walls, while on MWNTs (probably on SWNTs), the catalytic effect of metallic impurities is much lower.

The results here identify that there is a clear maximum in the electrocatalytic activity of residual metal impurities-blocked (i.e., metal-free) pristine CNTs for OER, HER and ORR as a function of number of walls of CNTs in alkaline solutions. The electrocatalytic activity of CNTs with 2-3 walls, i.e., CNT-2, CNT-3 and CNT-4 is significantly higher than typical SWNTs, CNT-1 and MWNTs, CNT-6 and CNT-7. Such distinctive volcano-type dependence of the activity of CNTs is neither related to the surface oxygen groups such as $\mathrm{C}-\mathrm{O}$, $\mathrm{C}=\mathrm{O}$ and $\mathrm{C}-\mathrm{OO}$ defects nor to the specific surface area. The oxygen groups on the outer walls of CNTs as determined by XPS vary in the range of 4.17 to $8.33 \%$ (Fig. S4, Table S2, ESI $\dagger$ ). For example, CNT-2 contains a $4.17 \%$ oxygen group, similar to $4.77 \%$ on CNT- 6 , however, CNT-2 shows a significantly higher activity, as compared with CNT-6. The activity of CNTs cannot be explained based on the surface areas of CNTs (see Table S1 and Fig. S6, ESI $\dagger$ ). Both theoretical and experimental investigations have indicated that the affinity of CNTs with the water molecule or the $\mathrm{OH}$ group increases with the decrease in the diameter of CNTs due to the increased misalignment of $\pi$-orbitals. ${ }^{15-18}$ Therefore, the increased electrocatalytic activity from CNT-1 to CNT-3 cannot be ascribed to the increase of size of CNTs.

The significant reduction in the electrocatalytic activities of CNTs and complete poisoning of $3.5 \mathrm{wt} \%$ NiCo loaded CNT-7 as compared to pristine CNT-7 (Fig. S5, ESI $\dagger$ ) in $1 \mathrm{M} \mathrm{KOH}+10 \mathrm{mM}$ KCN solutions demonstrates that KCN is capable of completely blocking the catalytic activities of residual metal impurities within CNTs. However, despite the significantly reduced activity, the CNTs for OER, HER and ORR show almost identical volcano curves as a function of the number of walls. This clearly indicates that the substantially high catalytic activity of CNTs with 2-3 walls is not solely contributed by the metallic impurities within CNTs. Therefore we argue that the distinctive volcano-shaped activity curves for OER, HER and ORR on CNTs are a clear indication of intrinsic properties of CNTs and the critical role of the inner tubes on the electrocatalytic activity of CNTs. Fujisawa et al. studied the electrical properties of SWNTs and DWNTs and showed experimentally that the inner tubes contribute significantly to the electronic transport properties of DWNTs. ${ }^{19}$ The exact mechanism for the high electrocatalytic activity of CNTs with 2-3 walls is not fully understood at this stage. However, the most likely reason for the high activity of DWNTs and TWNTs (i.e., CNT-2, CNT-3 and CNT-4) is the effective separation of the functionality of the outer wall and inner tubes; the outer wall provides reaction sites for the adsorption and dissociation of $\mathrm{OH}^{-}$ads, $\mathrm{O}_{2 \text {,ads }}, \mathrm{O}_{\text {ads }}$ species for the electrochemical reactions, while the protected inner-tubes serve as the effective electronic conducting pathway for the charge transfer process of the reaction. The effective electron transfer between the outer wall and inner tubes could occur through electron tunnelling under the electrochemical polarization driving force, similar to the electron tunnelling through thin oxide films as proposed by Damjanovic et al. for the OER on $\mathrm{Pt}^{20}$ The distance between the 


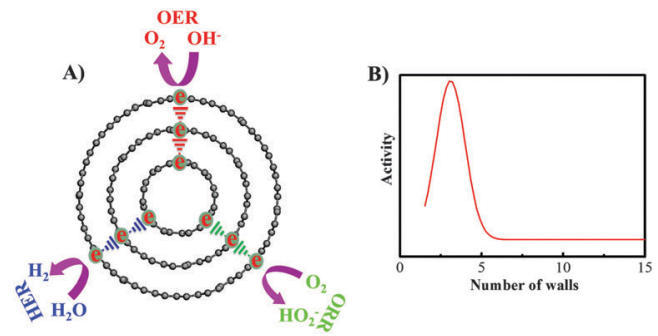

Fig. 4 (A) Scheme of electron charge transfer steps for OER, HER and ORR through electron tunnelling effect on DWNTs and/or TWCNTs; (B) generalized electrocatalytic activity of CNTs as a function of the number of walls.

outer-most wall and the inner tubes is $\sim 0.32 \mathrm{~nm},{ }^{21}$ which is in the range of that for electron tunnelling. ${ }^{22}$ Fig. 4 shows schematically the role of the effective electron transfer between the outer wall and inner tubes on the OER, HER and ORR on CNTs with 2-3 walls.

Evidently, such separation of the functionality and electron transfer between the outer wall and inner tubes would not be possible for SWNTs. The proposed mechanism implies that the electron transfer between the outer wall and inner tubes would strongly depend on the distribution of the driving force, the applied polarization potential across the inner tubes. In the case of MWNTs, the driving force of polarization potential for the electron transfer or tunnelling between the outer wall and inner tubes would decrease with the increase in the number of inner tubes. Thus, the electron transfer or tunnelling between the outer surfaces to the inner tubes could become less favourable as the number of walls/layers increases. This hypothesis explains why double/triple-walled CNT-2, CNT-3 and CNT-4 are far more active for the electrochemical reactions than MWNTs, CNT-6 and CNT-7. As shown early, ${ }^{10}$ the CNTs are inherently structurally and chemically stable for the electrochemical reactions in alkaline solutions, a significant advantage as compared to the nano-structured based electrocatalysts.

The electrochemically active species for OER is $\mathrm{OH}^{-}$, while for ORR and HER, it is $\mathrm{O}_{2}$ and $\mathrm{H}_{2} \mathrm{O}$ molecules, respectively, as shown below:

$$
\begin{gathered}
\text { OER: } 4 \mathrm{OH}^{-} \rightarrow \mathrm{O}_{2}+2 \mathrm{H}_{2} \mathrm{O}+4 \mathrm{e} \\
\text { ORR: } \quad \mathrm{O}_{2}+\mathrm{H}_{2} \mathrm{O}+2 \mathrm{e} \rightarrow \mathrm{HO}_{2}^{-}+\mathrm{OH}^{-} \\
\text {HER: } \quad 2 \mathrm{H}_{2} \mathrm{O}+2 \mathrm{e} \rightarrow \mathrm{H}_{2}+2 \mathrm{OH}^{-}
\end{gathered}
$$

The origin of the overpotential or barrier of an electrochemical reaction can be directly linked to the proton and/or electron transfer to the adsorbed reaction species bonded to the surface of the electrocatalysts, which in turn depends on the interaction energy between the species and the catalyst surface. As shown by Norskov et al. for the ORR, that of Pt is very close to optimum and metals that have either stronger or weaker bonding of oxygen than Pt are poor ORR catalysts. ${ }^{23}$ The interaction energy of water on the CNT surface is very weak due to the inert nature of CNTs and the extension of the nanotubes have modest effect on the interaction energies. ${ }^{24}$ The weak bonding of water molecules indicates that barrier for HER would be large. This is evidently confirmed by the very high Tafel slopes of $210-400 \mathrm{mV} \mathrm{dec}{ }^{-1}$ for HER on CNTs (Fig. S7, ESI $\dagger$ ), which is substantially higher than $74 \mathrm{mV} \mathrm{dec}^{-1}$ observed on $\mathrm{Co}^{25}$ and $75 \mathrm{mV} \mathrm{dec}^{-1}$ on Pt in alkaline solutions. ${ }^{26}$ ORR on CNTs is similar to that on glassy carbon and most likely proceeds by two-electron transfer process with the formation of hydrogen peroxide intermediates (Fig. S8, ESI $\dagger$ ). In the case of OER in alkaline solutions, the discharge of the $\mathrm{OH}^{-}$ species would be relatively easy due to the high affinity of $\mathrm{OH}^{-}$ions on CNTs, and hence, kinetically OER is more favourable on the surface of the CNTs ${ }^{10}$ as compared to ORR and HER (Fig. S9, ESI $\dagger$ ).

In summary, the results show that the metal-free (i.e., impurity-blocked) pristine CNTs are electrocatalytically active, consistent with that reported by Byers on SWNTs, ${ }^{9}$ but strongly dependent on the number of walls or inner tubes of CNTs. Such intrinsic activity of CNTs is most likely related to the quantum properties of CNTs, i.e., the significantly enhanced charge transfer via the electron tunnelling between the outer wall and inner tubes via electron tunnelling of CNTs under the electrochemical polarization potential driving force. The residual metallic impurities do play an important role; however, their electrocatalytic activity is most pronounced on CNTs with 2-3 walls.

This work was financially supported by the Australian Research Council under Discovery Project Scheme (project number: DP150102044), Australia.

\section{Notes and references}

1 J. Wu, Y. Xue, X. Yan, W. Yan, Q. Cheng and Y. Xie, Nano Res., 2012, $5,521-530$

2 S. Y. Wang, S. P. Jiang, T. J. White, J. Guo and X. Wang, J. Phys. Chem. C, 2009, 113, 18935-18945.

3 C. Liu and H. M. Cheng, Mater. Today, 2013, 16, 19-28.

4 I. Kruusenberg, N. Alexeyeva, K. Tammeveski, J. Kozlova, L. Matisen, V. Sammelselg, J. Solla-Gullon and J. M. Feliu, Carbon, 2011, 49, 4031-4039.

5 I. Kruusenberg, N. Alexeyeva and K. Tammeveski, Carbon, 2009, 47, 651-658.

6 I. Kruusenberg, J. Leis, M. Arulepp and K. Tammeveski, J. Solid State Electrochem., 2010, 14, 1269-1277.

7 P. H. Matter, E. Wang, M. Arias, E. J. Biddinger and U. S. Ozkan, J. Phys. Chem. B, 2006, 110, 18374-18384.

8 L. Wang and M. Pumera, Chem. Commun., 2014, 50, 12662-12664.

9 J. C. Byers, A. G. Guell and P. R. Unwin, J. Am. Chem. Soc., 2014, 136, $11252-11255$.

10 Y. Cheng, C. W. Xu, L. C. Jia, J. D. Gale, L. L. Zhang, C. Liu, P. K. Shen and S. P. Jiang, Appl. Catal., B, 2015, 163, 96-104.

11 W. Yuan, Y. Cheng, P. K. Shen, C. M. Li and S. P. Jiang, J. Mater. Chem. A, 2015, 1961-1971.

12 J. Zhang, Y. Cheng, S. Lu, L. Jia, P. K. Shen and S. P. Jiang, Chem. Commun., 2014, 50, 13732-13734.

13 S. Gupta, C. Fierro and E. Yeager, J. Electroanal. Chem., 1991, 306, 239-250. 14 M. S. Thorum, J. M. Hankett and A. A. Gewirth, J. Phys. Chem. Lett., 2011, 2, 295-298.

15 D. A. Britz and A. N. Khlobystov, Chem. Soc. Rev., 2006, 35, 637-659. 16 Z. F. Chen, W. Thiel and A. Hirsch, ChemPhysChem, 2003, 4, 93-97. 17 S. Niyogi, M. A. Hamon, H. Hu, B. Zhao, P. Bhowmik, R. Sen, M. E. Itkis and R. C. Haddon, Acc. Chem. Res., 2002, 35, 1105-1113.

18 A. Bilic and J. D. Gale, J. Phys. Chem. C, 2008, 112, 12568-12575.

19 K. Fujisawa, K. Komiyama, H. Muramatsu, D. Shimamoto, T. Tojo, Y. A. Kim, T. Hayashi, M. Endo, K. Oshida, M. Terrones and M. S. Dresselhaus, ACS Nano, 2011, 5, 7547-7554.

20 A. Damjanovic, V. I. Birss and D. S. Boudreaux, J. Electrochem. Soc., 1991, 138, 2549-2555.

21 S. Iijima, Nature, 1991, 354, 56-58.

22 C. Li, E. T. Thostenson and T.-W. Chou, Appl. Phys. Lett., 2007, 91, 223114.

23 J. K. Norskov, J. Rossmeisl, A. Logadottir, L. Lindqvist, J. R. Kitchin, T. Bligaard and H. Jonsson, J. Phys. Chem. B, 2004, 108, 17886-17892.

24 P. Schyman and W. L. Jorgensen, J. Phys. Chem. Lett., 2013, 4, 468-474. 25 S. P. Jiang and A. C. C. Tseung, J. Electrochem. Soc., 1991, 138, 1216-1222. 26 B. V. Tilak and C. P. Chen, J. Appl. Electrochem., 1993, 23, 631-640. 